\title{
Fabrication of all-metal field emitter arrays with controlled apex sizes by molding
}

\author{
E. Kirk, ${ }^{\text {a) }}$ S. Tsujino, ${ }^{\text {b) }}$ and T. Vogel \\ Laboratory for Micro- and Nanotechnology, Paul Scherrer Institut, CH-5232 Villigen-PSI, Switzerland \\ K. Jefimovs \\ Electronics/Metrology/Reliability Laboratory, EMPA, Überlandsstrasse 129, CH-8600 Dübendorf, \\ Switzerland \\ J. Gobrecht \\ Laboratory for Micro- and Nanotechnology, Paul Scherrer Institut, CH-5232 Villigen-PSI, Switzerland
}

A. Wrulich

PSI-XFEL, Paul Scherrer Institut, CH-5232 Villigen-PSI, Switzerland

(Received 23 October 2008; accepted 13 May 2009; published 23 June 2009)

\begin{abstract}
The authors proposed a method to fabricate field emitter arrays with uniform apex diameters in tens of nanometer scale based on the molding technique and apply it to fabricate molybdenum field emitter arrays. Apex diameter equal to $23 \pm 5 \mathrm{~nm}$ was observed in a $6 \times 6$ tip array by high-resolution scanning electron microscope. They also studied the field-emission characteristics in devices with gate electrodes fabricated on top of the arrays by a self-aligned process. In single-gate devices, emission current of up to $20 \mu \mathrm{A}$ per tip with negligible gate leak current was observed. The gate-fabrication process was extended to fabricate double-gated emitters. Further optimization of the fabrication process for higher emission current, together with metallurgical and lithographic methods, is discussed. (C) 2009 American Vacuum Society. [DOI: 10.1116/1.3151852]
\end{abstract}

\section{INTRODUCTION}

A double-gate field emitter array (FEA) device equipped with a focusing gate, in addition to an extraction gate, is potentially advantageous over the state-of-the-art photocathode for applications that require extreme low emittance and high brightness, such as the cathode for a compact free electron laser (FEL) with subnanometer wavelength. ${ }^{1,2}$ According to the $x$-ray FEL (XFEL) design developed by the PSIXFEL project at the Paul Scherrer Institut, an XFEL with significant reduction in the size and cost can be realized using a cathode with emission current above $5 \mathrm{~A}$ with $10-40 \mathrm{ps}$ duration having the normalized emittance below $0.1 \mathrm{~mm}$ mrad with a total emission area of less than $1 \mathrm{~mm}$ in diameter. $^{1,2}$ The single-gate, Spindt-type FEA cathode ${ }^{3}$ has been applied to vacuum-tube $\mathrm{rf}$ amplifiers. ${ }^{4}$ However, the total current is limited to $\sim 0.3 \mathrm{~A}^{5}$ Double-gate FEAs are not available commercially.

Unlike the abovementioned Spindt-type FEAs, the allmetal, double-gate FEAs that we are developing are fabricated by a molding technique. The Spindt-type FEAs consist of conical shape molybdenum emitters fabricated after the other structural layers by evaporation of the molybdenum through the gate-aperture holes, while controlling the aperture hole size in situ. In the molding technique, originally proposed by Gray and Green ${ }^{6}$ for FEAs, the emitters are fabricated before the other structural layers by the deposition of the emitter material into molds prefabricated in a substrate and subsequent removal of the substrate. The gate electrodes

${ }^{\text {a) }}$ Electronic mail: eugenie.kirk@psi.ch

${ }^{b)}$ Electronic mail: soichiro.tsujino@psi.ch are patterned afterward by a self-aligned gate process using planarization and etch back. ${ }^{7}$ The reported fabrication of molybdenum FEAs by the molding technique used $\mathrm{Si}$ wafer molds. ${ }^{8} 9$ There, the application of crystal orientation dependent wet-etching produces pyramidal-shaped mold pits with uniform apex sizes. The lining of such molds by thermally grown $\mathrm{SiO}_{2}$ produces a cusp-shaped apex and side ridges. ${ }^{9}$ In this way, the apex radius can be of the order of $1 \mathrm{~nm}$ or less, which is advantageous to lower the turn-on gate voltage. However, the cusp-shaped side ridges lead to the distortion of the gate-aperture holes as well as parasitic emission that can cause bombardment of the gate electrode and premature breakdown of the device.

In mold-based FEAs, one can exploit the wide range of tip materials to produce tips whose smoothness, sharpness, and reproducibility depend only on the mold characteristics with the use of standard semiconductor processes and equipment. ${ }^{9}$ Fabrication of comparatively large area arrays is possible, particularly in combination with hot-embossing technology. ${ }^{10}$ Compared to molded FEAs, control of the tip shape is more difficult when completed emitters are coated to provide a range of tip materials. ${ }^{11}$ Materials with low electron affinity, greater emission stability, longer lifetime, and other desirable properties were deposited into molds, either before the metal to make a surface layer on the tip, or in some cases replacing the metal entirely. Due to properties including low electron affinity and high thermal conductivity, diamond rapidly became the material of most interest. ${ }^{12-18}$ $19 \mathrm{~mA}$ emission current was achieved from ungated diamond FEAs with $10^{7}$ tips and total emitter area of $\sim 1 \mathrm{~cm}^{2}{ }^{16}$ Other materials reported were $\mathrm{WSi}^{19}{ }^{19} \mathrm{LaB}_{6}$, and $\mathrm{TiN},{ }^{11}$ 
$\mathrm{SiC}^{20}{ }^{20} \mathrm{NbN},{ }^{21}$ and modified photoresist. ${ }^{10}$ These were studied for flat panel displays ${ }^{22}$ and also to produce tips for atomic force microscopy and scanning tunneling microscopy $^{23}$ and for scanning near-field optical microscope. $^{24}$

The addition of the second gate to form a vertical triode structure for focusing operation was demonstrated experimentally in $1995 .^{25}$ In double-gate FEAs, loss of anode current, either by suppression of field emission from the tip or repulsion of electrons into the extraction electrode, could present a problem, depending on the strength of the focusing field. Methods to electrically screen the tip included positioning it below the gate ${ }^{26}$ or increasing the extractor gate thickness. $^{27}$ To reduce the repulsive effect, it was proposed ${ }^{28}$ to use a much larger diameter focusing aperture, partway to an in-plane scheme despite the disadvantage of reduced tip density. Local and out-of-plane focusing electrodes have been fabricated for most types of FEA, including $\mathrm{Si}$ wafer-molded $^{29}$ and cusp-molded emitters, ${ }^{30}$ Spindt-type emitters, ${ }^{31}$ etched Si emitters, ${ }^{27,25,32}$ and carbon nanotube (CNT) emitters. ${ }^{33}$ Elimination of cross-talk in field emitter displays $^{31}$ and sources for multibeam electron beam lithography ${ }^{23}$ were seen as potential applications. A 2007 review of the status of field emitter displays ${ }^{34}$ included three designs using local out-of-plane focusing, two Spindt-type and one CNT-type, which are close to commercial realization.

Rather than Spindt-type FEAs that have conical emitters or FEAs based on carbon nanotubes ${ }^{35}$ with cylindrical shape, we explore the field-emission properties of pyramidal-shaped molybdenum FEAs with metallic substrates based on the molding technique. Although to date the applications of molded metallic FEAs for high current are rare, the broaderbased pyramidal-shaped emitter tip should be advantageous because of the higher thermal conductance and thermal spread. $^{36}$ The low intrinsic resistivity of $\sim 5 \times 10^{-6} \Omega \mathrm{cm}$ and the high thermal conductivity of $1.4 \mathrm{~W} \mathrm{~cm}^{-1} \mathrm{~K}^{-1}$ of molybdenum at room temperature are also advantageous for the thermal stability of the tips at high emission current. The metallic substrate is potentially advantageous over $\mathrm{Si}$ substrates for short pulse operation when the device structure and the operations are optimized. To further optimize for high current, we developed a novel mold-shaping method to tailor the emitter apex curvature at the nanometer scale. Using this method, we optimize the emitter apex curvature for resistance to electromigration and melting at the tip apex to improve the single-tip current.

\section{FABRICATION PROCESS FOR MOLDING-BASED EMITTER ARRAY AND GATED DEVICES}

The schema in Fig. 1 show our fabrication steps for the emitter arrays, single-gate FEAs, and double-gate FEA structures. The process is based on the Si wafer molding technique and self-aligned gate process. ${ }^{8}$ Fabrication starts by preparation of Si molds, which consist of arrays of pyramidal pits in the surface of the wafers [Figs. 1(a) and 1(b)]. This is done by anisotropic wet etching of silicon using $20 \% \mathrm{KOH}$

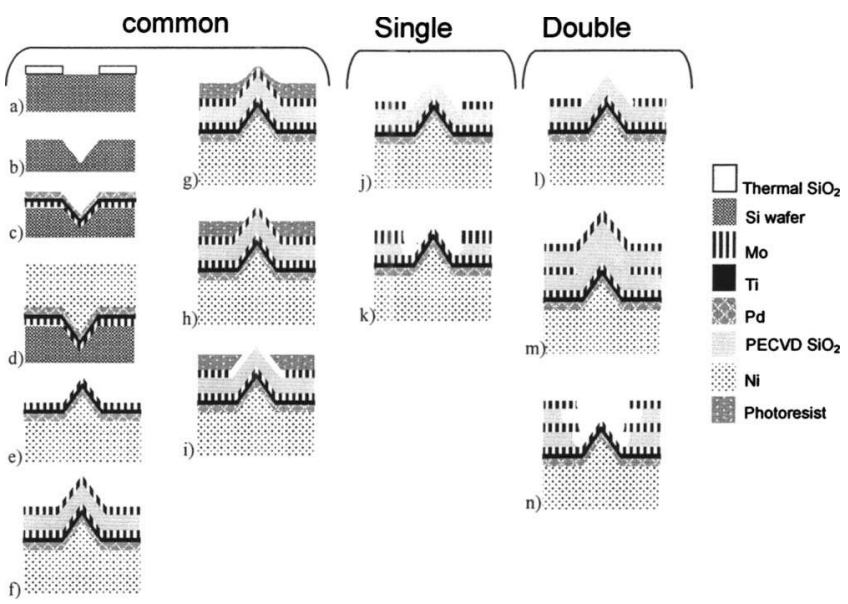

FIG. 1. Schematic representation of the FEA fabrication process: (a)-(i) show the steps common to the single- and double-gate processes. (j) and (k) show completion of the single-gate process, (1)-(n) show completion of the double-gate process.

solution in de-ionized water at $70{ }^{\circ} \mathrm{C}$. As the etching mask, we patterned a thermal $\mathrm{SiO}_{2}$ film grown on the wafer surface. The oxide mask is removed after etching using $7 \%$ hydrofluoric acid solution (buffered oxide etch, or BOE) [Fig. 1(b)]. Next we oxidize the mold wafer to grow a $\mathrm{SiO}_{2}$ layer, which protects the emitter material surface in a later step, during the chemical removal of the silicon mold wafer. The oxidation of the silicon mold also changes the shape of the molds; the bottom of the mold becomes cusp shaped which results in a needle-shaped emitter apex with extremely small curvature of the order of $1 \mathrm{~nm} .{ }^{11}$ The degree of elongation depends on the oxidation time. We remove the first oxide lining with BOE and oxidize the mold wafer a second time.

To make the emitters, $1-\mu \mathrm{m}$-thick Mo is deposited by sputtering into the oxidized mold. The base pressure of our sputtering system is $4 \times 10^{-6}$ mbar. At the Ar pressure of 1.6 mbar, the sputtering current of 1.5 A gives a Mo film, which, measured on the wafer surface outside the molds, is close to stress-free. Large sputter targets $\left(100 \times 200 \mathrm{~mm}^{2}\right)$ reduce the problem of shadowing within the molds across the 4 in. wafer. The Mo emitters are backed by $500-\mu$ m-thick Ni that serves as the substrate of the devices [Fig. 1(c)]. The Ni substrate was deposited by electroplating. As a seed layer for the electroplating, we deposited 100-nm-thick $\mathrm{Ti}$ and 200-nm-thick Pd on top of the Mo film. After the Ni electroplating [Fig. 1(d)], the mold wafer is removed by $\mathrm{KOH}$ etching, followed by the removal of the thermal oxide lining by 7\% BOE etching [Fig. 1(e)].

To fabricate gated devices, layers of insulator $[1 \mu \mathrm{m} \mathrm{SiO}$ deposited by plasma-enhanced chemical vapor deposition (PECVD)] and gate metal (500 nm sputtered Mo) are deposited on top of the demolded array [Fig. 1(f)]. The self-aligned process to pattern the aperture holes of the gate combines the etch back of a polymer layer by $\mathrm{O}_{2}$ plasma etching and wet etching of the Mo and $\mathrm{SiO}_{2}$ layers [Figs. 1(g)-1(k)]. We used photoresist layers of various thicknesses as the polymer for 
the planarization. In the process for the single-gate devices, Mo is etched from the aperture hole by a phosphoric acidbased wet etch using backetched photoresist as the mask [Fig. 1(i)]. After that, the photoresist is removed by $\mathrm{O}_{2}$ plasma etching and the $\mathrm{SiO}_{2}$ layer is etched from the aperture hole by $7 \%$ BOE using the Mo gate layer as an etch mask [Figs. 1(j) and $1(\mathrm{k})$ ]. The patterning of the gate contact by phosphoric acid-based wet etch completes the process.

To fabricate double-gate FEA structures, we first pattern the first gate layer by wet etching [Fig. 1(1)] and then the second insulator and gate are deposited [Fig. 1(m)]. The aperture holes of the second gate are patterned by the selfaligned process described above. Now the double thickness of insulator is cleared from the through hole to expose the tip [Fig. 1(n)]. Finally the gate contacts are defined by wet etching. We note that although the use of oxygen plasma is critical at some steps of the fabrication process, the exposure of the tip surface to the oxygen plasma causes a detrimental degradation of the field-emission properties. Therefore we have designed the process flow to avoid the direct exposure of the tips to $\mathrm{O}_{2}$ plasma.

\section{RESULTS AND DISCUSSIONS}

\section{A. Microstructure of sputtered Mo in mold holes}

To fabricate emitter tips, we deposited Mo by sputtering at room temperature. Good topographical coverage can be achieved by the sputtering technique. Nevertheless, inside the emitter molds, the Mo thickness on the walls tapers off with depth. When the deposited thickness of the Mo film is $1 \mu \mathrm{m}$ on the flat surface, the film thickness at the bottom of the mold pit is $\sim 600 \mathrm{~nm}$ [Fig. 2(b)]. We found from $\mathrm{x}$-ray diffraction study that our room temperature sputtered Mo is textured with (110)-orientation perpendicular to the substrate surface and has a columnar structure. Therefore, when the film grows on the mold walls and converges, large grains shadow smaller ones below them, preventing uniform film deposition. Because of this, complete filling of tips with high wall angles is difficult, in particular, when the bottom of the mold pits is extremely cusp shaped. As shown in Fig. 2(a), the deposited molybdenum forms a thin-walled tube at the tip of the mold. Therefore, to fabricate emitters which can carry high current, it is necessary to reduce the sharpness of the bottom of the mold pits intentionally. We indeed find that using the mold which has less pronounced cusp-shaped pits the filling of the mold is improved, as shown in Fig. 2(b). However, these tips still contain pores, particularly along the central axis of the mold pits.

We also studied the internal structure of the molybdenum film for a completed emitter by sputter etching using a focused ion beam microscope. In Fig. 3, we show the scanning electron microscopy (SEM) image of the cross section of the completed emitter. The position of the cross section is $10-100 \mathrm{~nm}$ in front of the emitter apex. We found that there are some pores in the Mo film as suggested in the cleaved cross section of Mo-coated molds in Fig. 2. The interface between the Mo and the $\mathrm{Ti} / \mathrm{Pd} / \mathrm{Ni}$ appears wavy in the cross

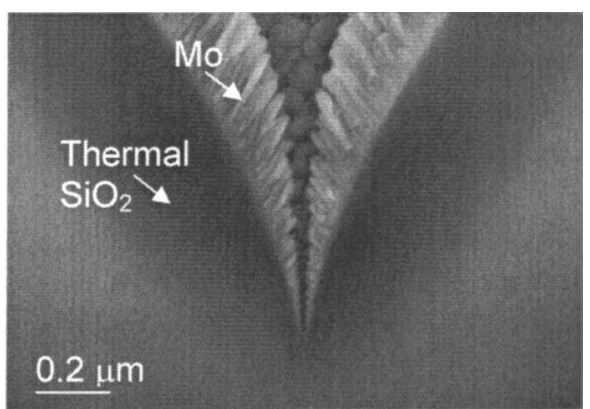

a)

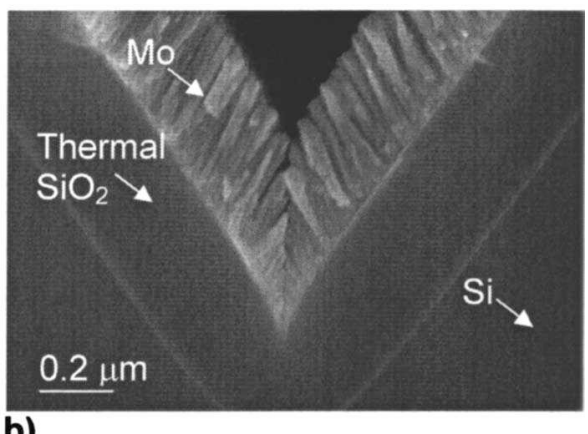

b)

FIG. 2. Cross sections through the emitter tips of two singly oxidized and Mo-coated Si molds with (a) $1 \mu \mathrm{m}$ and (b) $0.3 \mu \mathrm{m}$ thermal $\mathrm{SiO}_{2}$, showing the variation in tip elongation with oxide thickness. The Mo sputtering process cannot completely fill tip (a).

section. We consider this is because $\mathrm{Ti} / \mathrm{Pd} / \mathrm{Ni}$ layers conform to the Mo surface, which becomes increasingly rough inside the mold because of the shadowing during the deposition process.

We found that the mold filling by Mo can be improved by deposition at lower background pressure with higher substrate temperatures. Figure 4 shows the cross section through the tip of an emitter mold when the Mo was sputter deposited at the substrate temperature of $550{ }^{\circ} \mathrm{C}$. The background pressure was $2.5 \times 10^{-8}$ mbar. This tip is densely filled with the Mo film consisting of grains that are factors of 10-100 larger than the films shown in Fig. 2. In terms of improved electrical and heat conduction, sputter-depositing pure Mo at a temperature sufficient for diffusion and grain growth therefore looks promising. However, when the filled mold is

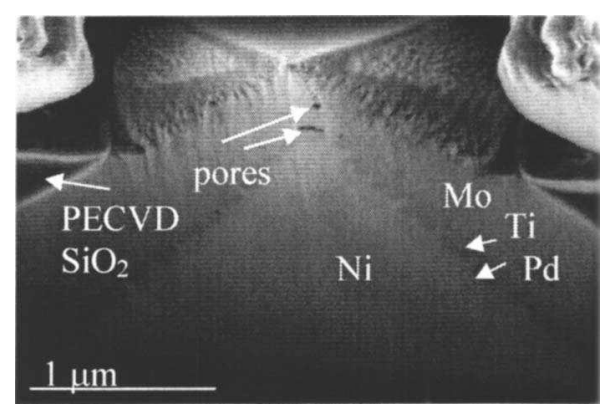

FIG. 3. Cross section of single emitter prepared by the focused ion beam. The emitter apex is visible at approximately $100 \mathrm{~nm}$ behind the cross section. Features visible in the cross section are labeled. The SEM image was taken from the tilt angle equal to $67^{\circ}$ from the sample normal direction. 


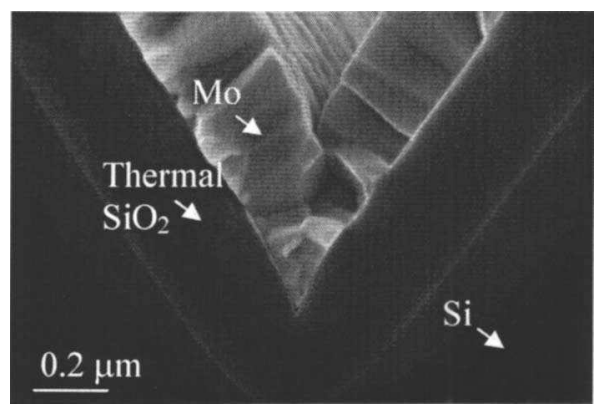

FIG. 4. Cross section through one emitter tip of a singly oxidized Si mold sputter coated with $\mathrm{Mo}$ at $550^{\circ} \mathrm{C}$. At this temperature, the sputter system had a base pressure of $2.5 \times 10^{-8}$ mbar. Large Mo grains and more dense tip filling, compared to room temperature sputter coating, are visible.

cooled to room temperature, it is under stress due to differential thermal contraction of the $\mathrm{Mo}$ and $\mathrm{Si}$ that causes cracks in the Mo film. The optimization of the deposition time, temperature, and film thickness to see whether there is a set of conditions that gives densely filled tips, together with a low enough thermal stress, is one of the focus of the next steps of the process development.

\section{B. Emitter shape control}

For a single-oxidation mold with the oxide thickness of $\sim 400 \mathrm{~nm}$, the shape at the bottom of the mold is moderately cusped [Fig. 2(b)]. Therefore, we can fill such a mold reasonably well and fabricate a FEA. The tip radius of such emitters is $\sim 5 \mathrm{~nm}$. However, the ridges of the mold pits where the facets of the pyramids join are also cusped and sharpened, increasing the probability of parasitic electron emission and breakdown in gated devices. When the oxide is thicker, the sharpness of the cusp-shaped mold pits is further enhanced. This results in a needlelike tip. Such an extremely sharp tip is advantageous for having high field enhancement and low-operation voltage. ${ }^{16}$ However, in the high current operation, those sharp tips are not suitable because the required current density is too high, while the heat sinking along the long shank is inefficient. Additionally, it is also difficult to coat the tip of such a sharp mold by sputtering as presented earlier [Fig. 2(a)].

The double-oxidation method is advantageous to control the mold shape to overcome these problems. For doubleoxidation molds, we remove the first oxide (oxide 1) with BOE. The Si cavity is now larger than the original mold, and all sharp features become smooth and concave. A second oxidation produces a mold whose facets meet in smooth curves. The tip cusping and sharpness depend on the thicknesses of oxide 1 and the second oxide (oxide 2).

In Fig. 5 we show the comparison of the completed and demolded individual emitters fabricated by double-oxidation mold [Figs. 5(a) and 5(b)] with oxide 1 and oxide 2 both $400 \mathrm{~nm}$ in thickness, and by single-oxidation mold with $400 \mathrm{~nm}$ oxide thickness [Figs. 5(c) and 5(d)]. The emitter fabricated by the single-oxidation mold indeed has sharp apex and sharp ridges. The double-oxidation tip [Figs. 5(a) and $5(\mathrm{~b})]$ is both more cusped and broader, ending in a

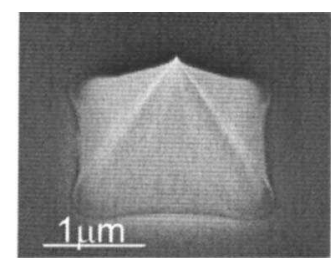

a)

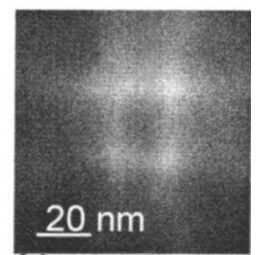

b)

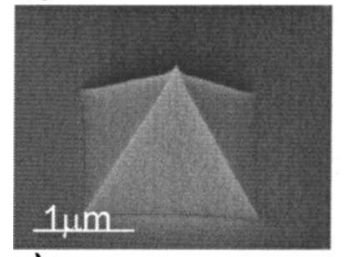

c)

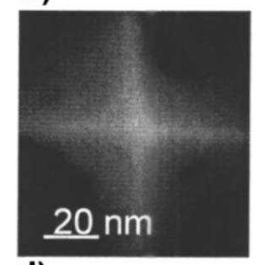

d)

FIG. 5. (a) Mo emitter from a doubly oxidized Si mold with first and second thermal $\mathrm{SiO}_{2}$ layers both $400 \mathrm{~nm}$. (b) Tip of the emitter viewed from above in the SEM. (c) Mo emitter from a singly oxidized Si mold with $400 \mathrm{~nm}$ thermal $\mathrm{SiO}_{2}$. (d) Tip of the emitter viewed from above in the SEM.

square platform [Fig. 5(b)]. Apex diameter equal to $23 \pm 5 \mathrm{~nm}$ was observed in a $6 \times 6$ tip array by highresolution scanning electron microscope (Fig. 6). This tip looks promising both for higher current and heat transport, while the increased cusping may improve field enhancement. The curved joins between facets reduce parasitic electron emission and the probability of arcing.

\section{Shape of the gate aperture}

The $\mathrm{Si}$ wafer molding process is attractive for the uniformity and sharpness of tips. However, the square-based pyramidal shape, in combination with self-aligned patterning, does not produce a circular aperture. It is not clear how a
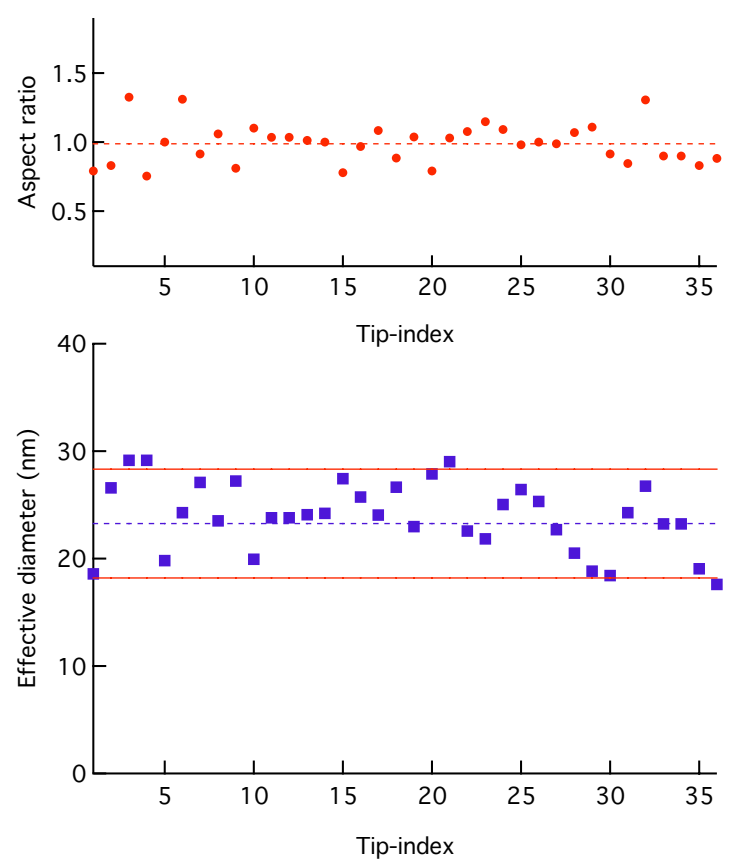

FIG. 6. (Color online) Size and aspect ratio of the apex of a $6 \times 6$ tip array measured by high-resolution scanning electron microscopy. 


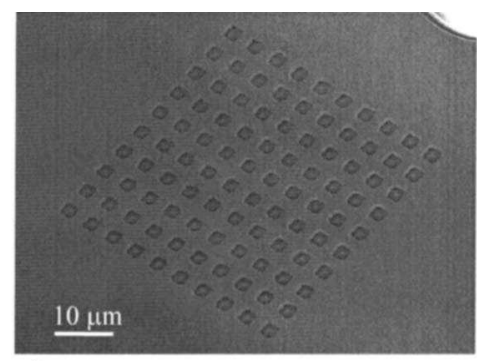

a)

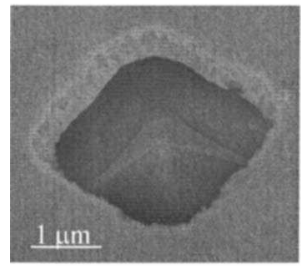

b)

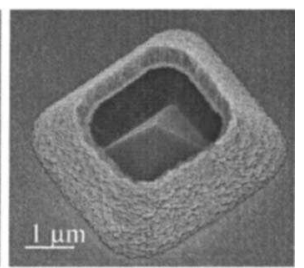

c)

FIG. 7. (a) Single-gated FEA test array, emitter tips, and extractor aperture coplanar, emitter height equal to insulator thickness. (b) One emitter in the array seen through the collarless aperture. (c) One emitter in an array where the emitter height is greater than the insulator thickness, showing the collar needed for coplanar tip and aperture.

squared-off aperture will affect the emittance of the electron beam. A further benefit of double-oxidation molding is that the curved joins between pyramid facets give a somewhat rounder aperture.

For emitters that are taller than the combined insulator plus gate metal, the aperture requires a raised collar, otherwise the tip will protrude through it, beyond the strongest extraction field. For shorter emitters, the collar is not needed. Figure 7(a) shows a single-gated, double-oxidation molded array of small emitters. A single emitter from the array [Fig. 7(b)] is compared to a larger emitter with collar [Fig. 7(c)]. For double-gated arrays, flat apertures on the first gate are desirable. The resist backetch for the second gate apertures is simpler without additional topography caused by a collar within the layers.

The self-aligned process can pattern flat, collar-free apertures if the resist is thinned to a few hundred nanometers, followed by an anisotropic overetch to remove the final part of the collar beneath the resist.

The shape and size of the apertures depend on the thickness of $\mathrm{SiO}_{2}$ and gate layers coating the emitter. For total overlayer thickness less than the emitter height, the surface profile above the emitters is approximately a square-based pyramid, enlarged by the overlayers and with extra $\mathrm{SiO}_{2}$ deposited on sharp features such as the tip. As the overlayer thickness increases, the surface profile evolves toward a hemisphere. Therefore the focusing aperture will be larger and more circular than the extractor aperture, depending on the thickness of the second $\mathrm{SiO}_{2}$ layer. This relationship may be used to achieve the desired aperture shape and diameter.

\section{Single-gate FEA structures}

Single-gate FEAs from three different wafers, A, B, and $\mathrm{C}$, were compared for their emission characteristics. The Mo

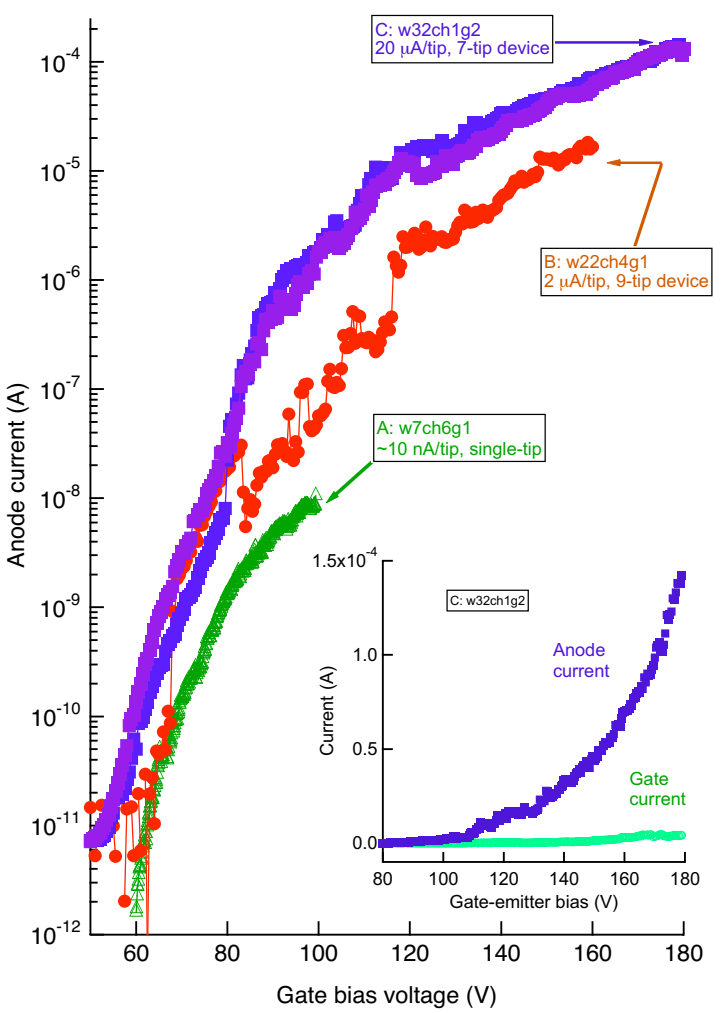

FIG. 8. (Color online) Current-voltage characteristics of three devices with molybdenum single-gate field emitters; A: w7ch6g1 (single tip), B: w22ch4g1( $3 \times 3$ tips), and C: w32chlg2 (7 tips). For A and B, the molybdenum film consisting the emitter were deposited on the mold fabricated by single-oxidation process, while it was deposited on the double-oxidation mold for device $\mathrm{C}$. The thickness of the emitter molybdenum film was $200 \mathrm{~nm}$ for device A, $500 \mathrm{~nm}$ for device B, and $1 \mu \mathrm{m}$ for device C.

emitters for A and B were deposited in single-oxidized molds with $400 \mathrm{~nm}$ oxide thickness, while $\mathrm{C}$ was deposited in a double-oxidized mold. Oxide 1 and oxide 2 were both $300 \mathrm{~nm}$. The emitter base length is $3 \mu \mathrm{m}$ and the height is $2.1 \mu \mathrm{m}$. The array pitch is $5 \mu \mathrm{m}$. The insulator is a $1.2-\mu \mathrm{m}$-thick silicon dioxide film deposited by PECVD and the gate layer is a $0.5-\mu \mathrm{m}$-thick molybdenum film deposited by sputtering at room temperature. The gate apertures and emitter tips were coplanar so the gated emitters have the collared structure seen in Fig. 7(c) which shows the SEM image of an emitter from the FEA C. Shipley 1828 resist was used for self-aligned patterning and was thinned by oxygen plasma etching in a reactive ion etcher (RIE) until holes with approximate side length of $2.5 \mu \mathrm{m}$ were opened over the emitters. Molybdenum was etched for $3 \mathrm{~min}$ and $20 \mathrm{sec}$ in a mixture of $76 \%$ phosphoric acid, $3 \%$ nitric acid, $15 \%$ acetic acid, and 6\% de-ionized water. Oxygen plasma etching in an RIE was used to remove the photoresist mask. The molybdenum apertures have side length of about $2.8 \mu \mathrm{m}$, including the overetch to remove the collar beneath the photoresist mask. The silicon dioxide underneath the aperture hole was removed by wet etching using 7\% BOE with 3 min etching time.

In Fig. 8, we show the current-voltage characteristics of FEAs A, B, and C. The number of tips in the array varied as 


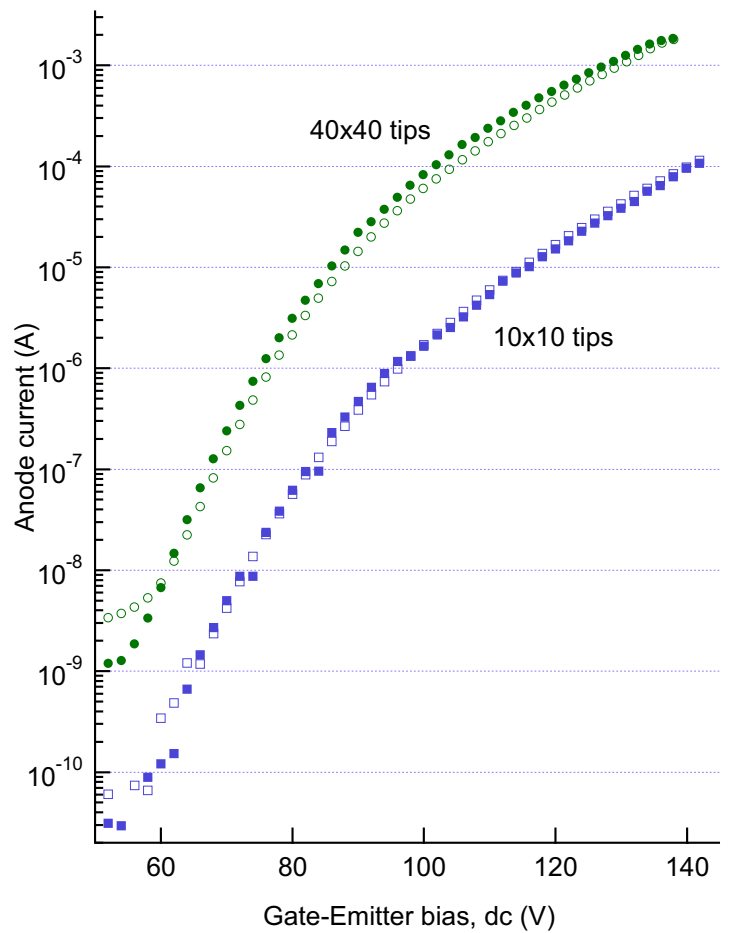

FIG. 9. (Color online) Comparison of the emission current from a $10 \times 10$ array device and a $40 \times 40$ array device depicted in Figs. 7(a) and 7(b). The hysteresis of the emission current between the increasing bias (filled signs) and the decreasing bias (empty signs) cases was minimal.

follows. A: single tip, B: $3 \times 3$ tips, and C: 7 tips. In these experiments, we continuously scanned the gate bias voltage between zero and a maximum bias voltage $V_{m}$ at the rate of $100 \mathrm{~V} \mathrm{~min}{ }^{-1}$ while gradually increasing $V_{m}$. The anode was held $\sim 10 \mathrm{~mm}$ apart from the arrays and was biased at $1 \mathrm{kV}$ dc. The background pressure of the measurement chamber was between $10^{-9}$ and $10^{-8}$ mbar. The displayed emission current-gate voltage characteristics were stationary after $2-4 \mathrm{~h}$.

We found that the maximum current was $\sim 10 \mathrm{nA}$ with $V_{m}$ of $\sim 100 \mathrm{~V}$ for device A but reached $\sim 140 \mu \mathrm{A}$ with $V_{m}$ of $180 \mathrm{~V}$ for device $\mathrm{C}$. We attribute the failure of device $\mathrm{A}$ at such limited current to inadequate Mo thickness: pinholes at joins in the structure allowed BOE to leak through during silicon dioxide etching, removing some of the subsurface layers, and weakening the structure. Such pinholes at the join are indicated in Figs. 2 and 3. A minimum thickness of $0.5 \mu \mathrm{m}$ Mo was found necessary for pinhole-free mold coating. Devices B and C had comparable numbers of tips, but as we have consistently found for the double-oxidation molded arrays, device $\mathrm{C}$ had higher emission current and was more stable. Emission current of up to $20 \mu \mathrm{A}$ per tip was observed for device $\mathrm{C}$. We attribute this to the improvements in emitter shape resulting from the double-oxidation mold process. The inset in Fig. 8 shows the current $I_{a}$ collected by the anode and the gate current $I_{\mathrm{g}}$ of device $\mathrm{C}$. We found that the emission current trapped by the gate electrode was small: $I_{\mathrm{g}}$ was below $3 \%$ of $I_{a}$.

Figure 9 compares the emission current from two FEAs fabricated from a fourth wafer D, a $10 \times 10$ array and a 40 $\times 40$ array. The wafer was deposited in a double-oxidized mold, with oxide thicknesses of $400 \mathrm{~nm}$ for oxide 1 and $500 \mathrm{~nm}$ for oxide 2 . The emitter base length is $2 \mu \mathrm{m}$ and the height is $1.2 \mu \mathrm{m}$. The array pitch is $5 \mu \mathrm{m}$. The insulator is a 1.2- $\mu \mathrm{m}$-thick silicon dioxide film deposited by PECVD and the gate layer is a $0.5-\mu \mathrm{m}$-thick molybdenum film deposited by sputtering at room temperature. Shipley 1813 resist was used for self-aligned patterning and was thinned by oxygen plasma etching in a reactive ion etcher until holes with approximate side length of $2 \mu \mathrm{m}$ were opened over the emitters. Etching of the molybdenum, removal of the photoresist mask, and etching of the oxide were the same as for wafers A-C. The molybdenum apertures on device D had a side length of about $2.5 \mu \mathrm{m}$, including the overetch to remove the collar beneath the photoresist mask. The completed $10 \times 10$ array is shown in Figs. 7(a) and 7(b). After $\sim 3$ days of continuous scan, as described above, the emission current became stable for both devices for the gate bias voltage range without exhibiting the sudden jump in emission current as observed in Fig. 8. At a given gate bias voltage, the ratio of the current is between 10 and 100, approximately equal to the ratio of the number of tips. This scaling of the current with the number of tips indicates the uniformity of the apex curvatures that was also shown by SEM characterization (Fig. 6). We also note that using a single-gate FEA shown here, an efficient laser-induced field-emission triggered by femtosecond near infrared light pulses was demonstrated recently. $^{37}$

\section{E. Double-gate FEA structures}

We produced double-gate FEA devices by extending the self-aligned gate process for the single-gate devices by following the process flow summarized in Fig. 1. In Fig. 10, we show a SEM image of a completed double-gate device based on the emitter array with $2 \mu \mathrm{m}$ base size and $1.2 \mu \mathrm{m}$ height. The emitters were fabricated using a double oxidation mold with the thickness of oxide 1 and oxide 2 both equal to $400 \mathrm{~nm}$. The thicknesses of both of the $\mathrm{SiO}_{2}$ insulator layers were equal to $1.2 \mu \mathrm{m}$. Both molybdenum gate layers were $0.5 \mu \mathrm{m}$ thick.

In order to preserve the $5 \mu \mathrm{m}$ pitch of the array, etch back of the S1813 photoresist mask for the extractor apertures was stopped as soon as the tips of the molybdenum were exposed. After wet etching for $3 \mathrm{~min}$ and $20 \mathrm{~s}$, the molybdenum apertures were close to circular, measuring approximately $1.6 \mu \mathrm{m}$ side length, with a collar therefore positioned above the tips. The second $\mathrm{SiO}_{2}$ layer and focusing gate layer were deposited, and the focusing apertures were patterned to a side length of approximately $2.6 \mu \mathrm{m}$. To remove the double thickness of $\mathrm{SiO}_{2}$ from the apertures, etching time was 6 min. The sidewalls of the lower $\mathrm{SiO}_{2}$ insulator, surrounding the clear tips, can be seen through the apertures in Fig. 10. In Fig. 11, we show the emission current $I_{\mathrm{em}}$ collected at the anode from this array when the emitter was biased at $-120 \mathrm{~V}$ and the focusing gate voltage, $V_{\mathrm{g} 2}$, was scanned between -60 and $60 \mathrm{~V}$ while holding the first gate 


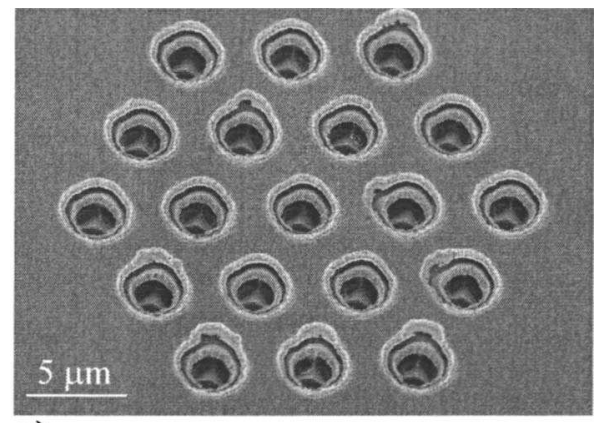

a)

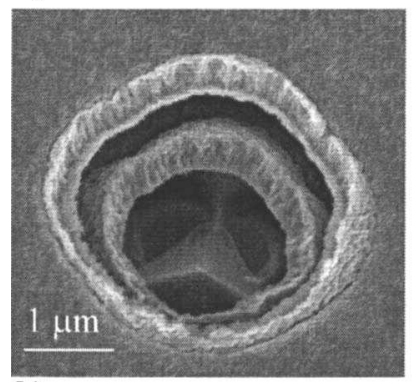

b)

FIG. 10. Double-gated field emitter array with $1.2 \mu \mathrm{m}$ tall, double-oxidation molded emitters. The pitch is less than $5 \mu \mathrm{m}$.

electrode to the ground potential. It confirms that this array functions as a double-gated device. When $V_{\mathrm{g} 2}$ is negative, the collimation function is expected. However, because the relationship between the tip, the extractor aperture, and the focusing aperture is not yet optimized, we observe that $I_{\mathrm{em}}$ varies sensitively with the change of $V_{\mathrm{g} 2}: I_{\mathrm{em}}$ was enhanced

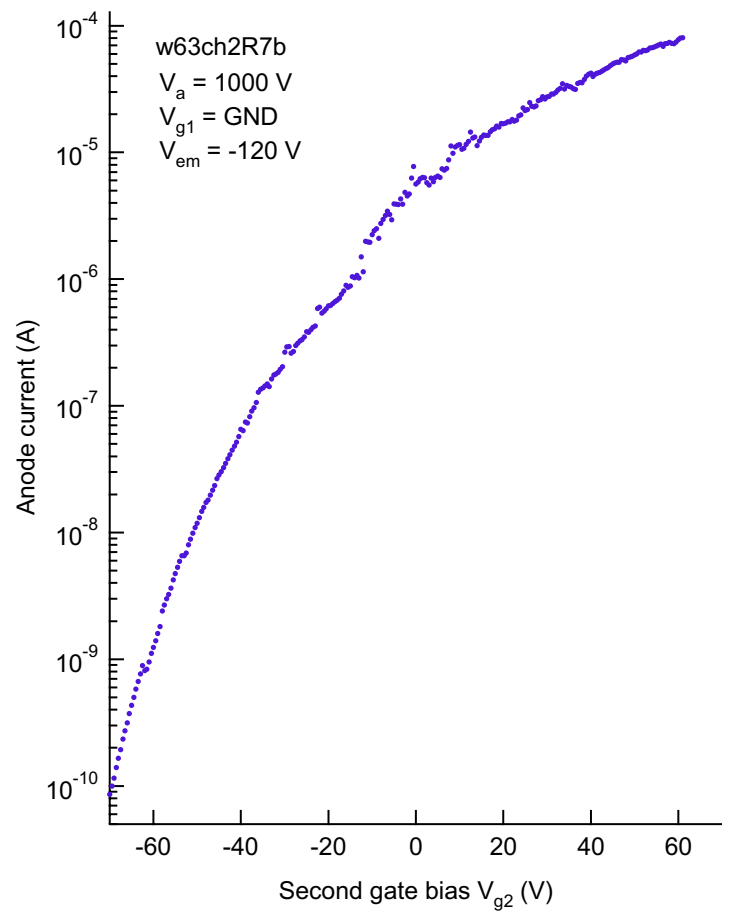

FIG. 11. (Color online) Current-voltage characteristics of the double-gate field emitter array device as a function of the second gate bias voltage when the emitter was biased at $-120 \mathrm{~V} \mathrm{dc}$. for positive $V_{\mathrm{g} 2}$ and $I_{\mathrm{em}}$ was reduced for negative values. Further research is underway to directly characterize the focusing function and to determine the layer thicknesses to optimize the focusing function.

\section{CONCLUSION}

We developed a fabrication process for all-metal FEA structures with single- and double-gate electrodes. By applying the double-oxidation process during mold manufacture, we have controllably increased the tip diameter of our emitters and removed the sharp ridges where facets join. A factor of 10 increased single-tip current, compared to singleoxidation molded emitters, resulted. Further increase in the emission current by the optimization of the tip shape and materials (for example, better Mo quality and microstructure) can be expected. Current density from the array will also be increased by using a smaller pitch, which can be achieved both by scaling down the entire structure (patterning the molds with e-beam lithography) and by developing an isotropic etch specific to the insulator layers.

\section{ACKNOWLEDGMENTS}

The authors wish to thank B. Haas, A. Weber, and R. Bischofberger for their precise and resourceful technical contributions to the field emitter fabrication.

${ }^{1}$ S. C. Leemann, A. Streun, and A. F. Wrulich, Phys. Rev. ST Accel. Beams 10, 071302 (2007).

${ }^{2}$ M. Dehler, A. E. Candel, and E. Gjonaj, J. Vac. Sci. Technol. B 24, 892 (2006).

${ }^{3}$ C. A. Spindt, J. Appl. Phys. 39, 3504 (1968).

${ }^{4}$ D. R. Whaley, B. M. Gannon, C. R. Smith, C. M. Armstong, and C. A. Spindt, IEEE Trans. Plasma Sci. 30, 998 (2002).

${ }^{5}$ P. R. Schwoebel, C. A. Spindt, and C. E. Holland, J. Vac. Sci. Technol. B 23, 691 (2005).

${ }^{6}$ Henry F. Gray and Richard F. Greene, U.S. Patent No. 4,307,507 (29 December 1981).

${ }^{7}$ Henry F. Gray and George J. Campisi, U.S. Patent No. 4,964,946 (23 October 1990).

${ }^{8}$ M. Sokolich, E. A. Adler, R. T. Longo, D. M. Goebel, and R. T. Benton, Tech. Dig. - Int. Electron Devices Meet. 1990, 159

${ }^{9}$ S. M. Zimmerman, D. B. Colavito, and W. T. Babie, Tech. Dig. - Int. Electron Devices Meet. 1990, 163.

${ }^{10}$ T. Asano, D. Sasaguri, E. Shibata, and K. Higa, Jpn. J. Appl. Phys., Part 1 36, 7749 (1997).

${ }^{11}$ M. Nakamoto, T. Hasegawa, T. Ono, T. Sakai, and N. Sakuma, Tech. Dig. - Int. Electron Devices Meet. 1996, 297.

${ }^{12}$ K. Okano, K. Hoshina, S. Koizumi, and J. Itoh, IEEE Electron Device Lett. 16, 239 (1995).

${ }^{13}$ W. P. Kang, J. L. Davidson, Q. Li, J. F. Xu, D. L. Kinser, and D. V. Kerns, Proceedings of the Eighth International Conference on Solid State Sensors and Actuators and Eurosensors IX Digest of Technical Papers, 1995 (unpublished), Vol. 2, pp. 182-185.

${ }^{14}$ A. Wisitsora-At, W. P. Kang, J. L. Davidson, M. Howell, W. Hofmeister, and D. V. Kerns, J. Vac. Sci. Technol. B 21, 1671 (2003).

${ }^{15}$ J. L. Davidson, W. P. Kang, and A. Wisitsora-At, Diamond Relat. Mater. 12, 429 (2003).

${ }^{16}$ K. Subramanian, W. P. Kang, J. L. Davidson, R. S. Takalkar, B. K. Choi, M. Howell, and D. V. Kerns, Diamond Relat. Mater. 15, 1126 (2006).

${ }^{17}$ Seongjin Kim, Byeong Kwon Ju, Yun Hi Lee, Beom Soo Park, YoungJoon Baik, Sunkyoo Lim, and Myung Hwan Oh, J. Vac. Sci. Technol. B 15, 499 (1997).

${ }^{18}$ N. Sakuma, T. Ono, and T. Sakai, IVMC'97 Technical Digest, 1997 (unpublished), pp. 451-454.

${ }^{19}$ T. Asano and J. Yasuda, Jpn. J. Appl. Phys., Part 1 35, 6632 (1996). 
${ }^{20}$ A. Gorecka-Drzazga, J. Dziuban, and E. Prociow, J. Vac. Sci. Technol. B 18, 1115 (2000).

${ }^{21}$ Y. Gotoh, Y. Kashiwagi, M. Nagao, T. Kondo, H. Tsuji, and J. Ishikawa, J. Vac. Sci. Technol. B 19, 1373 (2001)

${ }^{22}$ Byeong Kwon Ju, Seong Jin Kim, Yun Hi Lee, Beom Soo Park, Young Joon Baik, Sungkyoo Lim, and Myung Hwan Oh, Proceedings of the IVMC'96, 1996 (unpublished), pp. 530-533.

${ }^{23}$ T. Yagi, Y. Shimada, T. Ikeda, O. Takamatsu, K. Takimoto, and Y. Hirai, Trans. Inst. Electr. Eng. Jpn., Sect. E 117, 407 (1997).

${ }^{24}$ A. Gorecka-Drzazga, J. Dziuban, S. Bargiel, and E. Prociow, Meas. Sci. Technol. 17, 45 (2006).

${ }^{25}$ J. Itoh, Y. Tohma, K. Morikawa, and K. Shimizu, J. Vac. Sci. Technol. B 13, 1968 (1995).

${ }^{26}$ L.-Y. Chen and A. I. Akinwande, J. Vac. Sci. Technol. B 24, 1878 (2006).

${ }^{27}$ A. Hosono, S. Kawabuchi, S. Horibata, S. Okuda, H. Harada, and M. Takai, J. Vac. Sci. Technol. B 17, 575 (1999).

${ }^{28}$ C. Pye, J. Itoh, T. Hirano, and S. Kanemaru, IEEE Trans. Electron De- vices 44, 498 (1997)

${ }^{29}$ P. N. Minh, T. Ono, N. Sato, H. Mimura, and M. Esashi, J. Vac. Sci. Technol. B 22, 1273 (2004).

${ }^{30}$ Y. Lee, S. Kang, and K. Chun, J. Micromech. Microeng. 7, 332 (1997).

${ }^{31}$ L. Dvorson and T. Akinwande, SID Int. Symp. Digest Tech. Papers 30, 392 (1999).

${ }^{32}$ L. Dvorson, I. Kymissis, and A. Akinwande, J. Vac. Sci. Technol. B 21, 486 (2003).

${ }^{33}$ Jun Hee Choi et al., Technical Digest of the 17th International Vacuum Nanoelectronics Conference 2004 (unpublished), pp. 32-33.

${ }^{34} \mathrm{H}$. Mimura, Technical Digest of the Eighth IEEE International Vacuum Electronics Conference IVEC 2007, 2007 (unpublished), pp. 1-4

${ }^{35}$ W. I. Milne et al., J. Mater. Chem. 14, 933 (2004).

${ }^{36}$ W. W. Dolan, W. P. Dyke, and J. K. Trolan, Phys. Rev. 91, 1054 (1953).

${ }^{37}$ S. Tsujino, P. Beaud, E. Kirk, T. Vogel, H. Sehr, J. Gobrecht, and A. Wrulich, Appl. Phys. Lett. 92, 193501 (2008). 